\section{Du glycogène à la gouttelette lipidique}

\author{
Une intime connexion dans l'adipocyte brun
}

Alicia Mayeuf-Louchart, Hélène Duez
Université de Lille, Inserm, CHU Lille, Institut Pasteur de Lille, U1011-

Institut Européen de Génomique du Diabète (EGID),

1 rue du Pr Calmette, F-59019 Lille, France. alicia.mayeuf-louchart@inserm.fr

communs à tous les adipocytes (bruns et blancs), tels que Pparg (peroxisome proliferator-activated receptor $\gamma$ ) et Fabp4 (fatty acid-binding protein 4). Ce n'est qu'à partir du $16^{\mathrm{e}}$ jour de développement embryonnaire que le caractère génétique «brun » apparaît, avec notamment l'expression d'Ucpl, le marqueur caractéristique du tissu adipeux brun, essentiel à la fonction de thermogenèse. Des études de respirométrie réalisées ex vivo à partir de tissu adipeux brun isolé à différents stades du développement embryonnaire chez les souris ont montré que la chaîne respiratoire est découplée à partir du $17^{\mathrm{e}}$ jour de développement [5]. Si les adipocytes bruns possèdent, à ce stade, toutes les capacités pour produire de la chaleur, la difficulté d'accès au tissu adipeux brun de l'embryon, in utero, ne permet toutefois pas, à ce jour, de déterminer s'il en produit effectivement. $\varepsilon n$ revanche, il est fonctionnellement prêt à répondre à une exposition au froid, notamment lors de la naissance.

\section{Biogenèse des gouttelettes lipidiques dans l'adipocyte brun}

Les gouttelettes lipidiques sont des organites composés de triglycérides, entourés d'une membrane composée d'une monocouche de phospholipides, dans laquelle sont incorporées des protéines spécifiques, telles que celles de la famille des périlipines. Les gouttelettes lipidiques apparaissent à partir du $15^{\mathrm{e}}$ jour de développement embryonnaire dans l'adipocyte brun (Figure 1) [5]. En étudiant précisément cet organite en microscopie électronique, nous avons constaté que la gouttelette lipidique apparaît au sein 


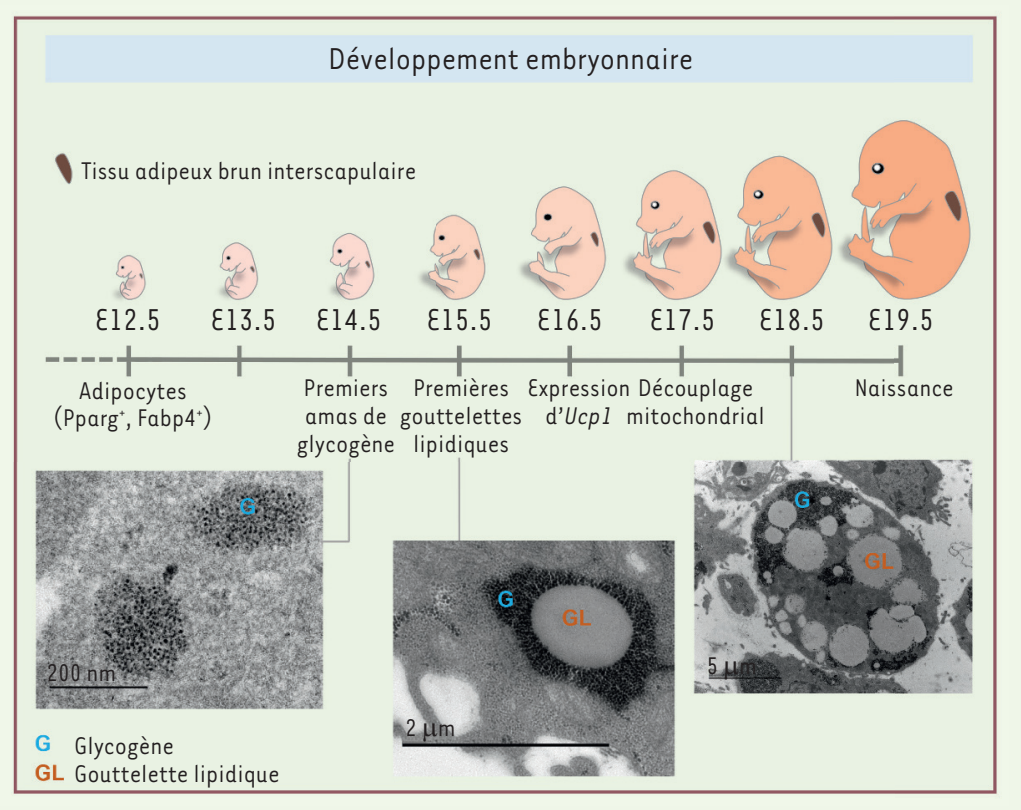

Figure 1. Différenciation du tissu adipeux brun au cours du développement embryonnaire chez la souris $(57 B / /$ J. Les premiers amas de glycogène $(G)$ apparaissent avant l'expression d'Ucpl dans l'adipocyte brun. Les gouttelettes lipidiques (GL) se forment au sein des amas de glycogène à partir du $15^{\mathrm{e}}$ jour de développement embryonnaire ( $\varepsilon$, embryonic day). Les gouttelettes lipidiques matures ne sont plus encerclées par du glycogène. Les images sont des photographies de microscopie électronique de tissu adipeux brun à différents stades du développement embryonnaire.

d'amas de glycogène dont la formation précède celle des gouttelettes lipidiques (Figure 1). Au cours du développement du tissu adipeux brun, de nouvelles gouttelettes lipidiques apparaissent au sein d'amas de glycogène nouvellement formés, et leur croissance est associée à une diminution de la quantité de glycogène qui les entoure. Cette association spatiale du glycogène et de la gouttelette lipidique avait été observée, dans les années 1960, dans le tissu adipeux brun de souris et de rats nouveau-nés [6, 7], mais depuis, aucune étude n'avait déterminé le rôle que pouvait jouer le glycogène dans ces cellules.

\section{Le glycogène essentiel à la formation des gouttelettes lipidiques}

Pour tenter de résoudre cette énigme, nous avons étudié les embryons de souris mutantes déficientes pour la glycogène synthase (souris Gys 1/-) [5]. Ces embryons, qui ne synthétisent pas de glycogène, ne présentent pas de gout- telettes lipidiques dans leurs adipocytes bruns, démontrant ainsi que le glycogène est essentiel à la formation de ces gouttelettes. Ces résultats ont été confirmés par des expériences d'interférence ARN ciblant la glycogène synthase dans des cellules humaines différenciées en adipocytes bruns in vitro.

Une analyse transcriptomique d'adipocytes bruns en cours de différenciation a permis de mettre en évidence l'augmentation de l'expression des gènes impliqués dans le métabolisme du glucose, l'activation du cycle de Krebs ainsi que la lipogenèse de novo, suggérant ainsi l'utilisation du glycogène au profit de la synthèse de nouveaux acides gras (Figure 2). La dégradation du glycogène s'effectue classiquement par des voies enzymatiques, dont la première étape est catalysée par la glycogène phosphorylase. Cependant, il existe un autre mécanisme, moins bien connu, de dégradation du glycogène: la glycophagie, c'est-à-dire l'autophagie du glycogène. La glycopha- gie a été décrite dans le foie, le muscle squelettique, et le cœur. Dans cette voie de dégradation du glycogène, la protéine Stbdl (Starch-binding domain-containing protein 1) s'associe au glycogène et est reconnue par la protéine Gabarapll (gamma-aminobutyric acid receptorassociated protein-like 1) localisée à la surface de l'autophagosome (Figure 2). Le glycogène est ainsi autophagocyté, puis dégradé en glucose 1-phosphate. Au cours de la différenciation des adipocytes bruns, la glycophagie joue un rôle important puisque des expériences d'ARN interférence ciblant le gène $S t b d l$ entraînent une diminution de la biogenèse des gouttelettes lipidiques. Le cycle de synthèse et de dégradation du glycogène est donc essentiel à la formation des gouttelettes lipidiques au cours de la différenciation de l'adipocyte brun. Qu'en est-il pour l'adipocyte blanc?

\section{Un rôle différent du glycogène dans l'adipocyte blanc?}

La plupart des études qui ont été effectuées pour identifier les mécanismes de formation de la gouttelette lipidique l'ont été dans l'adipocyte blanc. Ces études ont montré que les gouttelettes lipidiques se forment par bourgeonnement à partir du réticulum endoplasmique [8]. Alors que nos analyses en microscopie électronique ne montrent pas la présence du réticulum endoplasmique autour des gouttelettes lipidiques en formation au sein des amas de glycogène dans les adipocytes bruns, le bourgeonnement de la gouttelette lipidique à partir du réticulum endoplasmique est visible dans les adipocytes blancs en cours de différenciation chez l'embryon. Le glycogène est également présent dans ces cellules, mais il ne se localise pas autour des gouttelettes lipidiques, comme c'est le cas dans l'adipocyte brun. De même, l'absence de glycogène chez les embryons Gys $1^{-/-}$n'empêche pas la formation des gouttelettes lipidiques dans les adipocytes blancs. Ces données montrent donc que les mécanismes impliqués dans la biogenèse des gouttelettes lipidiques 


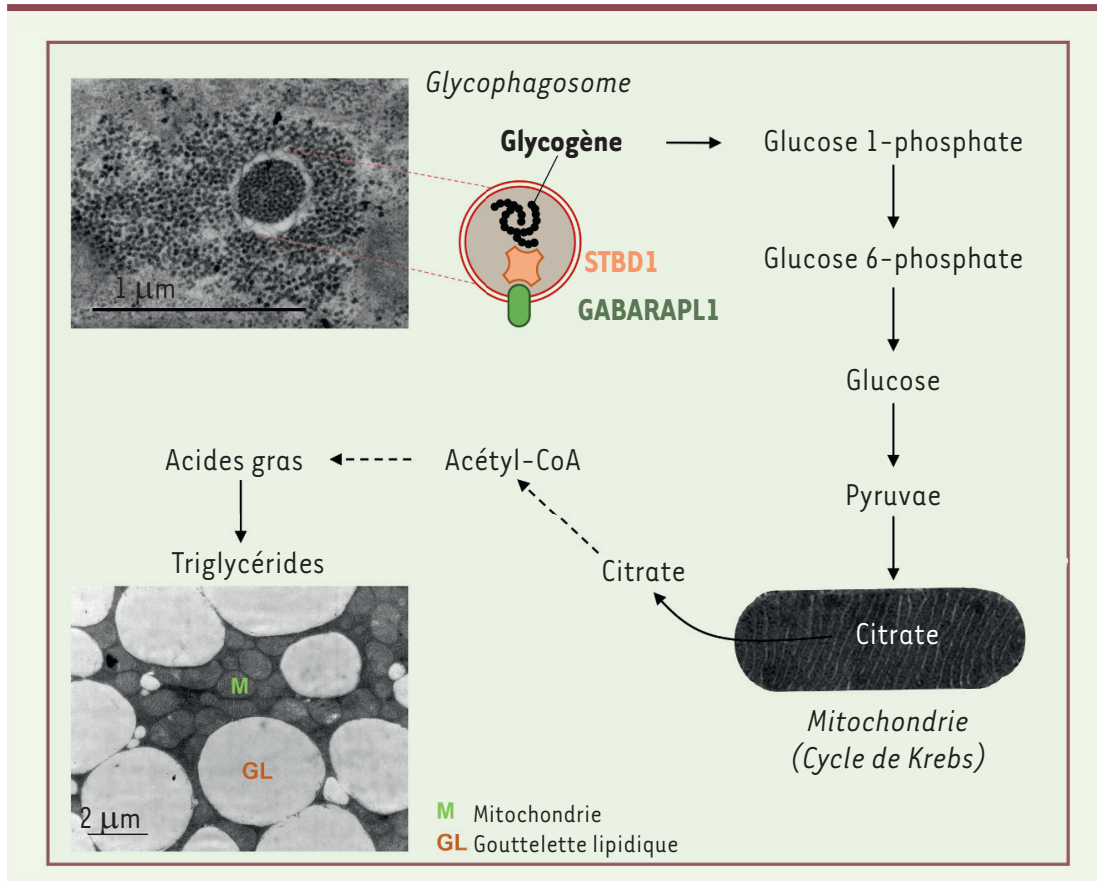

Figure 2. Voies métaboliques allant du glycogène aux triglycérides stockés dans les gouttelettes lipidiques. Le glycogène est dégradé par glycophagie dans des glycophagosomes, observables dans les adipocytes bruns au cours du développement embryonnaire, de $\varepsilon 15.5$ à $\varepsilon 18.5$. Les images sont des photographies de microscopie électronique du tissu adipeux brun.

sont différents entre adipocytes bruns et adipocytes blancs.

\section{Perspectives}

Jusqu'à présent, le glycogène et les gouttelettes lipidiques avaient été considérés comme des moyens de stockage énergétique indépendants. Nos travaux ont révélé l'existence d'une interaction spatiale et fonctionnelle dans l'adipocyte brun en cours de différenciation.
Dans le tissu adipeux brun chez l'adulte, une exposition au froid entraîne une accumulation transitoire de glycogène [9]. L'étude du métabolisme du glycogène dans les adipocytes du tissu adipeux brun chez l'adulte pourrait permettre d'identifier de nouvelles cibles thérapeutiques contre l'obésité. From glycogen to lipid droplet: an intimate connection in the brown adipocyte

\section{REMERCIEMENTS}

Cette recherche a bénéficié de soutiens financiers de l'ANR-labex-EGID (ANR-10-LABX-46), de la région des Hauts de France/FEDER (Chronoregeneration), de l'Association Française contre les Myopathies (AFM-Téléthon), de la Fondation de France et de la Fondation Francophone pour la recherche sur le diabète (FFRD), soutenu par la Fédération Française des Diabétiques (AFD), AstraZeneca, Eli Lilly, Merck Sharp \& Dohme (MSD), Novo Nordisk et Sanofi.

\section{LIENS D’INTÉRÊT}

Les auteures déclarent n'avoir aucun lien d'intérêt concernant les données publiées dans cet article.

\section{RÉFÉRENCES}

1. Bouloumié A, Sengenès C, Galitzky J. Les progéniteurs adipeux blancs et bruns : pourra-t-on transformer la fourmi en cigale? Med Sci (Paris) 2009 ; 25 : 123-5.

2. van Marken Lichtenbelt WD, Vanhommerig JW, Smulders NM, et al. Cold-activated brown adipose tissue in healthy men. $N$ Engl J Med 2009 ; 360 : 1500-8.

3. Carrière $A$, Jeanson $Y$, Cousin $B$, et al. Le recrutement et l'activation d'adipocytes bruns et/ou BRITE : une perspective réelle pour le traitement des maladies métaboliques? Med Sci (Paris) 2013 ; 29 : 729-35.

4. Wang W, Seale P. Control of brown and beige fat development. Nat Rev Mol Cell Biol 2016 ; 17 : 691702.

5. Mayeuf-Louchart A, Lancel S, Sebti Y, et al. Glycogen dynamics drives lipid droplet biogenesis during brown adipocyte differentiation. Cell Rep $2019 ; 29$ : 1410-8. e6.

6. Revel JP, Napolitano L, Fawcett DW. Identification of glycogen in electron micrographs of thin tissue sections. J Biophys Biochem Cytol $1960 ; 8$ : 575-89.

7. Napolitano L, Fawcett $D$. The fine structure of brown adipose tissue in the newborn mouse and rat. J Biophys Biochem Cytol 1958 ; 4 : 685-92.

8. Walther TC, Chung J, Farese RV. Lipid droplet biogenesis. Annu Rev Cell Dev Biol 2017 ; 33 : 491-510.

9. Jakus PB, Sandor A, Janaky T, et al. Cooperation between BAT and WAT of rats in thermogenesis in response to cold, and the mechanism of glycogen accumulation in BAT during reacclimation. J Lipid Res $2008 ; 49: 332-9$.






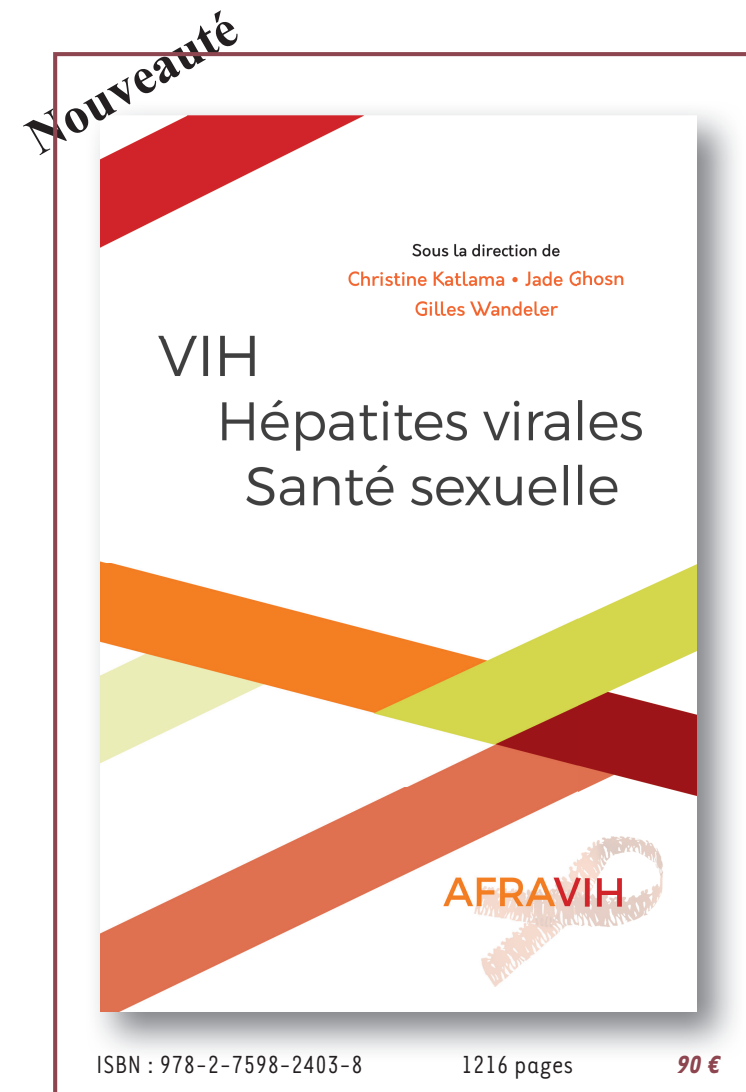

\section{Infection VIH}

Bientôt, plus de 40 millions de personnes à travers le monde vivront avec le VIH. Et la pandémie continue de s'aggraver. Pourtant, la riposte contre le VIH a profondément transformé la Santé publique, mobilisant acteurs de santé et communautés de patients, afin d'accélérer la recherche pour parvenir rapidement à des traitements accessibles. De dévastatrice, l'infection par le VIH est devenue une maladie chronique, qui, si elle est dépistée tôt et correctement prise en charge, permet de vivre en relative bonne santé. Pour autant, de nombreux efforts restent à faire, en termes de prévention et de dépistage, pour espérer pouvoir enrayer un jour cette maladie.

\section{Hépatites virales}

Près d'un Français sur 100 serait atteint d'une hépatite B ou C, et la moitié d'entre eux l'ignorerait. Longtemps méconnues car silencieuses, ces hépatites, non traitées, peuvent évoluer vers une cirrhose ou un cancer du foie. Nous disposons aujourd'hui de vaccins efficaces pour prévenir l'hépatite $B$, et d'antiviraux guérissant plus de $95 \%$ des personnes infectées par l'hépatite C. Pourtant, l'accès au diagnostic et au traitement doit être renforcé pour atteindre l'objectif ambitieux de l'Organisation mondiale de la Santé (OMS), d'éradication des hépatites B et C d'ici à 2030.

\section{Santé sexuelle}

Plus de quinze ans après la reconnaissance par l'OMS de la santé sexuelle comme partie intégrante de la santé, le contraste entre définition et pratiques reste saisissant. Parce que la sexualité appartient à notre quotidien, qu'elle contribue à notre bien-être et à notre qualité de vie, elle doit pouvoir être abordée librement, sans tabous et sans crainte auprès des professionnels de santé. Renforcer leur formation est indispensable, afin qu'ils puissent adapter leurs conseils, et diagnostic en fonction de chaque usager.

Cet ouvrage fait le point des connaissances, des stratégies et des programmes d'actions à conduire dans trois domaines clés de la santé publique, trois défis de dimension mondiale qui concernent l'ensemble des populations et dont l'intrication pose des questions de santé globale : la lutte contre le VIH et contre les hépatites, avec la prise en compte de la santé sexuelle comme élément déterminant de la santé.

Christine KATLAMA, professeure de Maladies Infectieuses, Sorbonne Université, praticien à l'AP-HP, Hôpital La Pitié-Salpêtrière, Paris ; présidente de I'AFRAVIH Jade GHOSN, maître de conférences à la Faculté de médecine de l'Université de Paris, praticien à l'AP-HP Nord, Hôpital Bichat-Claude Bernard, Paris Gilles WANDELER, professeur du Fonds National Suisse de Recherche, chef de clinique au Département des Maladies Infectieuses de I'Hôpital Universitaire de Berne en Suisse

À retourner à EDP Sciences, 17, Avenue du Hoggar - BP 112 - 91944 Les Ulis Cedex A - France Tél. : 33 (0) 169187575 - ع-mail : livres@edpsciences.org

NOM : .................................................... Prénom :.

Adresse:

Code postal : Ville :

Pays :

$\varepsilon$-mail : Tél :

Je souhaite recevoir VIH - Hépatites virales - Santé sexuelle : $90 €+10 €$ de port (France métropolitaine) = $100 €$ TC Hors France, merci de contacter expeditions@edpsciences.org pour obtenir les frais de port.

en ................. exemplaire, soit un total de ..... $€$

$\square$ Par chèque, à l'ordre de EDP Sciences

$\square$ Par carte bancaire : $\square$ Visa $\square$ Eurocard $\square$ American express

Carte $n^{\circ}$ I I I I I I I I I I I I I I I I I I I I

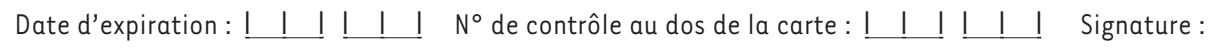

Ce livre est disponible en version numérique à l'adresse www.afravih.org 